Case report

\title{
The rarity of nongravid uterus torsion - difficult diagnosis even in modern medicine
}

\author{
Ludmila Stirbu ${ }^{1}$, Marius Lucian Savin ${ }^{2,3, *}$, Lidia Ionescu ${ }^{1,2}$, Radu Dănilă ${ }^{1,2}$, Delia \\ Gabriela Ciobanu Apostol ${ }^{2,4}$, Roxana Maria Livadariu ${ }^{1,2}$
}

${ }^{1} 3^{\text {rd }}$ Surgical Unit, "Sf. Spiridon" University Hospital, Iasi, Romania; "'Grigore T. Popa” University of Medicine and Pharmacy, lasi, Romania; ${ }^{3}$ Department of Radiology, "Sf. Spiridon" University Hospital, lasi, Romania; ${ }^{4}$ Department of Pathology, "Sf. Spiridon" University Hospital, lasi, Romania

\begin{abstract}
Uterine torsion is a rare surgical emergency that can evolve with ischemic lesions of the genital organs. We present a case of a 59 year-old woman who was hospitalized with important abdominal pain and modified general status. On clinical examination, a giant abdominal mass was found, and bimanual genital palpation could not find the cervix but revealed tension in the vaginal walls. CT examination described the palpable tumor mass as a giant uterine leiomyoma, with no information regarding the adnexes. As her general status become critical within 24 hours, the surgical intervention was performed, although the patient refused it. The abdominal exploration discovered a giant engorged uterus with 720-degree dextro-isthmic rotation around its axis; both fallopian tubes, broad ligaments, round ligaments and ovaries were also rotated and ischemic. She underwent subtotal hysterectomy with favorable postoperative outcome. The pathological report showed that the uterus and the annexes had ischemic to necrotic lesions. This case shows that the diagnosis of uterine torsion can be a pitfall due to its rarity. The disease has fatal evolution if the correct treatment is not applied on time.
\end{abstract}

Keywords: uterine torsion; nongravid; devolvulation; diagnostic pitfalls

\section{Introduction}

Uterine torsion is a rare surgical emergency. It may have a rapid evolution with ischemic lesions of the implicated internal genital organs, that will lead to acute and rapid clinical deterioration. The rarity of this condition makes it difficult to diagnose, both clinically and with imaging. We present a case of a 720-degree dextro-torsion of a leiomyomatous uterus along with the annexes in a 59 years old woman.

Received: x 2019; Accepted after review: x 2019; Published: September 2019.

${ }^{*}$ Corresponding author: Marius Lucian Savin, $3^{\text {rd }}$ Department of Radiology, "Sf. Spiridon" University Hospital, 1 Independentei Bvd., lasi, Romania;

Email:savinmarius@gmail.com

\section{Case report}

A 59 years old postmenopausal obese patient presented in emergency with important abdominal pain starting 2 weeks before, which increased in intensity in the last 2 days.

On clinical examination we found a giant abdominal tumor with its upper pole in the epigastrium. The mass was firm and painful on palpation. On bimanual examination, the cervix was unreachable, and the vaginal walls felt like in tension. The report after computed tomography (CT) investigation showed the presence of the giant uterine leiomyoma with important vascularization and the presence of a minimum quantity of free fluid in the left parauterin space, without giving any information regarding the annexes (Figures 1 and 2). 


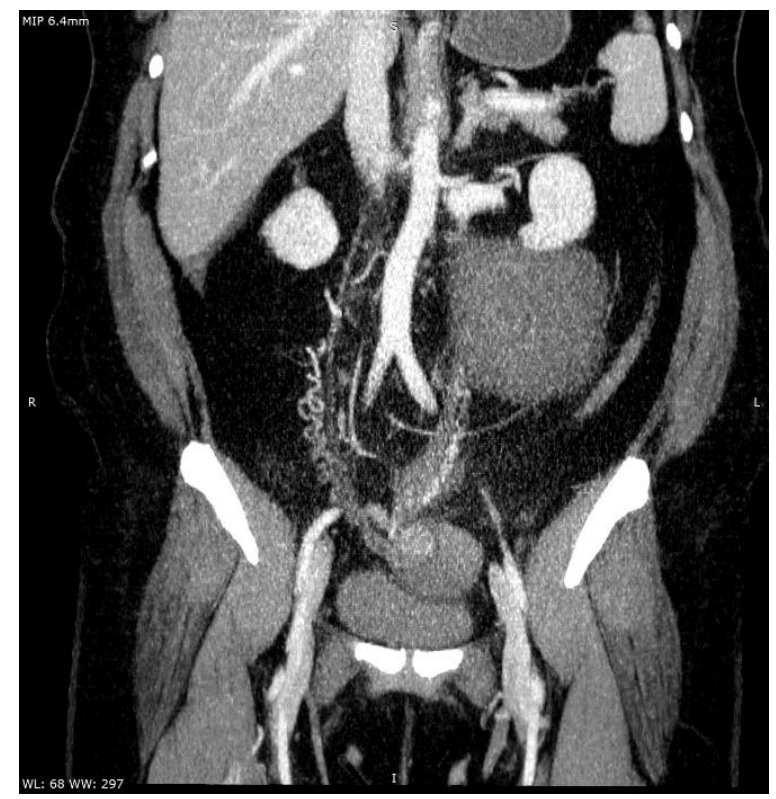

Fig. 1. CT Maximum Intensity Projection (MIP) showing serpiginous ovarian arteries converging in the region of the uterine isthmus

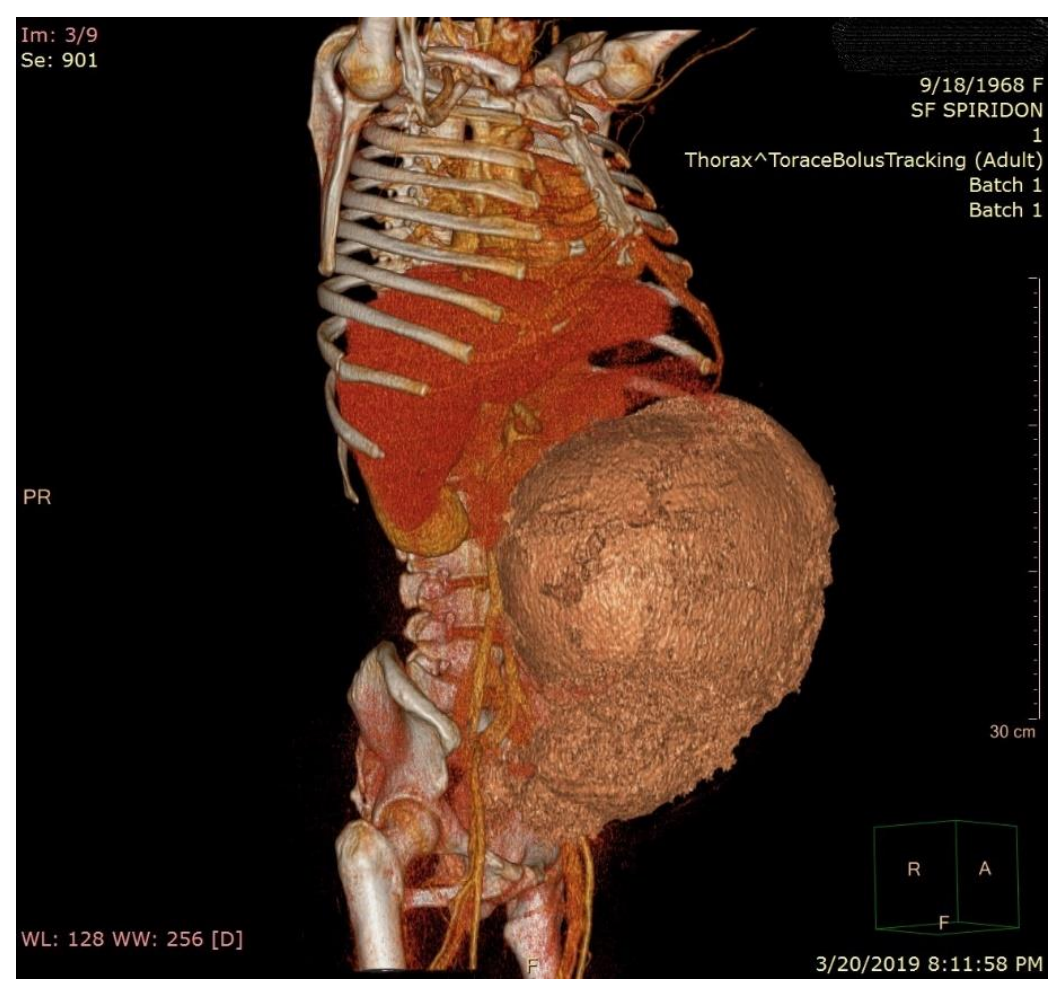

Fig. 2. CT aspect - Volumetric Rendering (VR) showing the uterine mass

The patient refused from the beginning any surgical intervention. Her general status became critical within the next 24 hours; she presented an episode of important pain on sudden mobilization, followed by faintness and important alteration of the general status with dyspnea, tachypnea, low blood pressure (systolic pressure $-80 \mathrm{~mm} \mathrm{Hg}$ ), tachycardia (160 bpm). Blood tests showed marked decrease of hemoglobin value from $10.2 \mathrm{mg} / \mathrm{dl}$ on admission to $6.5 \mathrm{mg} / \mathrm{dl}$, acidosis $(\mathrm{pH}=7.2)$ and increased lactate $(6.5 \mathrm{mmol} / \mathrm{L})$.

She underwent surgical intervention in emergency. Abdominal exploration revealed a 
giant engorged uterus with 720-degree dextro isthmic rotation around uterus axis; both fallopian tubes, broad ligaments, round ligaments and ovaries were also rotated and ischemic (Figures 3 and 4). After devolvulation, there were no improvements in the ischemic lesions of all mentioned organs. We proceeded with a subtotal hysterectomy and bilateral adnexectomy. We consider important to mention that the only permeable artery was the left uterine artery. We considered subtotal hysterectomy because, without having a preoperative diagnosis, we did not obtain the patient's informed consent for total hysterectomy before surgery. More, the operation was made in emergency, and a "damage control" procedure was preferred.

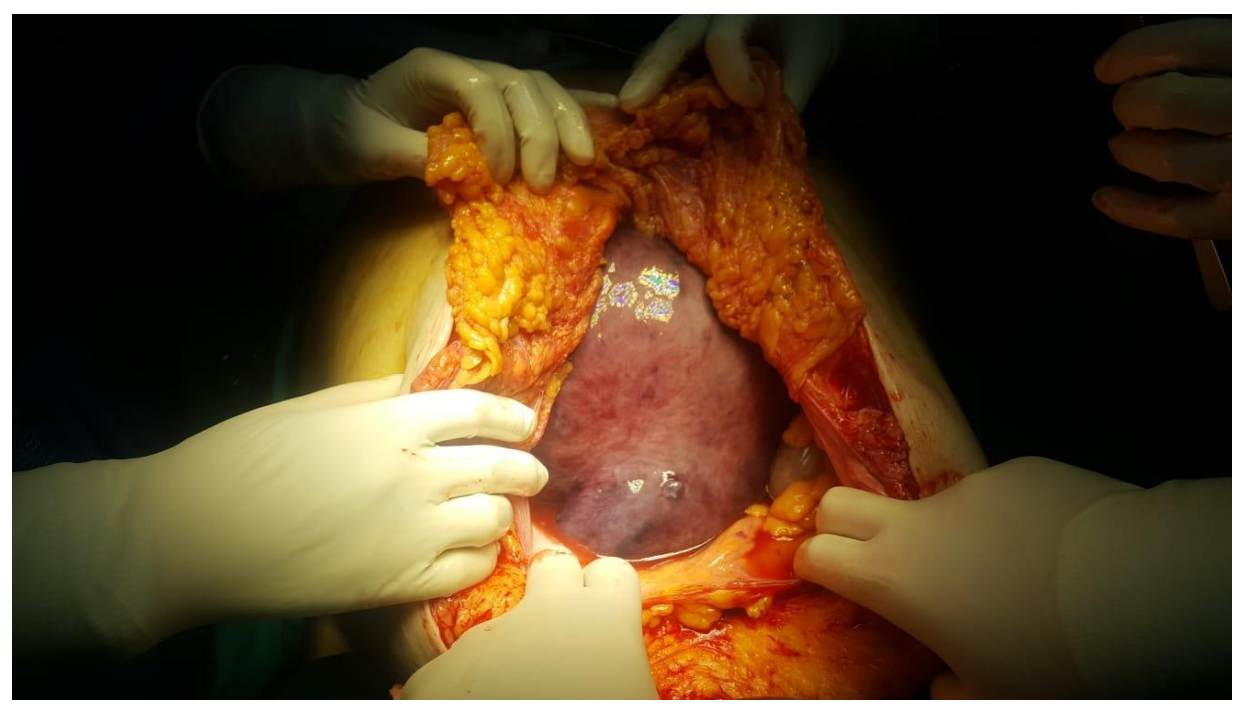

Fig. 3. Intraoperative aspect

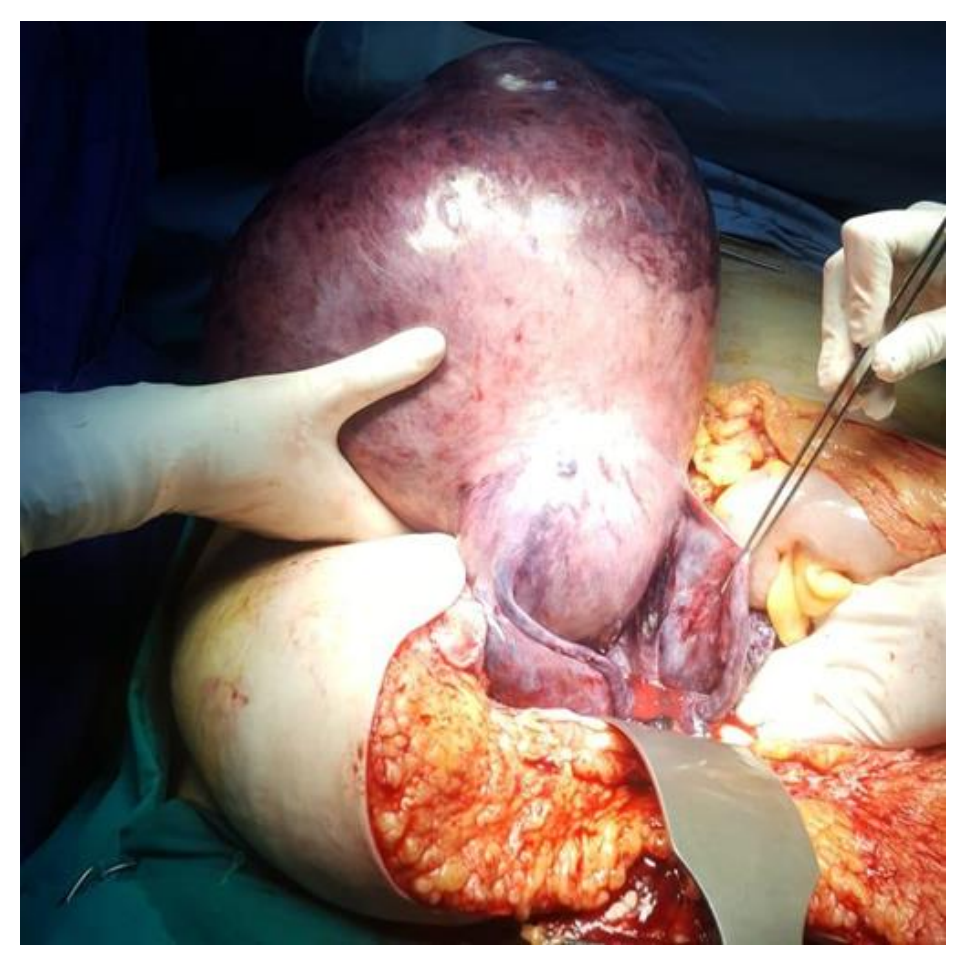

Fig. 4. Intraoperative aspect after devolvulation 
The postoperative evolution was simple with discharge on the $6^{\text {th }}$ day. The anatomopathological report showed that the posterior wall and the fundus of the uterus were occupied by a leiomyoma of $26 / 23 / 18 \mathrm{~cm}$ with features of infarction and important necrosis, with obliterative thrombosis of the intramural vessels. The endometrium had important ischemic lesions with quasicomplete hemorrhagic. The right adnexa had total necrosis with hemorrhage while the left adnexa had only important hemorrhagic suffusions. All these hemorrhagic lesions related to the torsion of the uterus and adnexae explain the important decrease in hemoglobin values in the last 24 hours of evolution, in condition of slightly modified coagulation parameters.

\section{Discussions}

The rotation of the uterus by more than 45 degrees up to 720 degrees defines uterine torsion [1].

Most commonly encountered in pregnant women, it develops around its long axis at the isthmic level; it may also arise in nongravid uterus when associated with pathological situations such as myomas, adnexal tumors, congenital abnormalities, pelvic adherences $[2,3]$.

Uterine torsion was first described in 1861 and until 2018 there were less than 50 cases of nongravid uterine torsion reported in literature $[4,5]$.

The uterine torsion is such a rare condition that even with modern imaging techniques, such as computed tomography, the diagnosis is usually missed before surgical exploration as it happened in our case also.

There are some specific clinical signs described, such as the impossibility of cervix visualization or distortion of the vagina and cervical canal on speculum examination, palpable anterior uterine artery upon pelvic examination, the cervix hitched under symphysis on vaginal examination or impossibility to palpate separately the uterine body $[1,6,7]$. Even so, the reduced clinical experience turns it into a diagnostic pitfall.

Our patient had some of the clinical signs that should have led towards the correct diagnosis. More, this patient benefited from contrast enhanced computed tomography in emergency, which only diagnosed the giant uterine mass. But the lack of experience concerning this specific pathology of the emergency team composed only of general surgeons and not by gynecologists, made the diagnosis possible only during surgery.

Worsening of the clinical status is due to vascular compromise consisting in venous obliteration that will result in uterine engorgement. Ischemic lesions may occur, and without emergency surgical intervention will lead to irreversible changes [6]. This is an important issue mostly in young women who want to preserve fertility. In our patient, intramural hemorrhage and tissue ischemia were reflected in biological changes (anemia with halving of the hemoglobin value in first 12 hours from presentation, decreased $\mathrm{pH}$, increased lactate) and in the general status of the patient (nonresponsive to the treatment for hypotension and shock).

\section{Conclusions}

The axial torsion of the nongravid uterus is a very rare but possible fatal medical condition. Due to its rarity, the theoretical knowledge that should help establish the diagnosis is lacking in nongynecological doctors.

\section{Consent}

Written informed consent was obtained from the patient for publication of this case report.

\section{Competing interests}

The authors declare that they have no competing interests. 


\section{References}

1. Jensen JG. Uterine torsion in pregnancy. Acta Obstet Gynecol Scand 1992; 71(4):260-265.

2. Luk SY, Leung JLY, Cheung ML, So S, Fung $\mathrm{SH}$, Cheng SC. Torsion of a nongravid myomatous uterus: radiological features and literature review. Hong Kong Med J 2010; 16(4):304-306.

3. Grover S, Sharma $Y$, Mittal S. Uterine Torsion: A Missed Diagnosis in Young Girls? J Pediatr Adolesc Gynecol 2009; 22(1):5-8.

4. Hawes $\mathrm{CH}$. Acute axial torsion of the uterus. Ann Surg 1935; 102(1):37-40.

5. Cheong EHT, Tan TJ, Wong KM. Torsion of a myomatous, non-gravid uterus: CT findings. $J$ Radiol Case Rep 2018; 12(4):6-14.

6. Sharma D, Usha MG. Torsion of a non-gravid uterus: a rare cause of acute abdomen. Int $J$ Reprod Contracept Obstet Gynecol 2013; 2(2):234-236.

7. Havaldar N, Ashok K. Torsion of non-gravid uterus with ovarian cyst - an extremely rare case. Pan Afr Med J 2014; 18:95. 